\title{
Parthenolide suppresses tumor growth in a xenograft model of colorectal cancer cells by inducing mitochondrial dysfunction and apoptosis
}

\author{
SE-LIM KIM, KIEU THE THU TRANG, SEONG HUN KIM, IN HEE KIM, \\ SEUNG OK LEE, SOO TEIK LEE, DAE GHON KIM and SANG-WOOK KIM \\ Department of Internal Medicine and Research Institute of Clinical Medicine, Chonbuk National \\ University Hospital, Chonbuk National University, Jeonju 561-712, Republic of Korea
}

Received May 14, 2012; Accepted July 9, 2012

DOI: 10.3892/ijo.2012.1587

\begin{abstract}
Parthenolide (PT), a principal active component in medicinal plants, has been used conventionally to treat migraine and inflammation. This component has recently been reported to induce apoptosis in cancer cells, through mitochondrial dysfunction. In the present study, we investigated PT-mediated cell death signaling pathway by focusing on the involvement of Bcl-2 family members in human colorectal cancer cells. We also investigated the inhibitory effect of PT on tumor growth in xenografts. Using the human colorectal cancer cell lines HT-29, SW620 and LS174T, we demonstrated that treatment of these cancer cells with PT induces apoptosis using MTT, Annexin V assay and Hoechst 33258 staining. Apoptosis through the mitochondrial pathway was confirmed by detecting regulation of Bcl-2 family members, cytochrome c release and caspase activation. Moreover, intraperitoneal injection of PT showed significant inhibition of tumor growth, angiogenesis in the xenograft model. These results demonstrate that PT exhibits anti-cancer activity in human colorectal cancer in vitro and in vivo. These findings may also provide a novel approach for the treatment of colorectal cancer.
\end{abstract}

\section{Introduction}

Colorectal cancer (CRC) is one of the most common malignancies worldwide (1). Other than surgery, treatment of CRC patients relies primarily on chemotherapy, especially the patients with advanced CRC. Among the chemotherapeutic agents for CRC, 5-fluorouracil (5-FU), which is a classical chemotherapy

Correspondence to: Dr Sang-Wook Kim, Department of Internal Medicine, Chonbuk National University Hospital, 634-18 Geumam2-dong, Duckjin-gu, Jeonju 561-712, Republic of Korea

E-mail: clickm@jbnu.ac.kr

Key words: parthenolide, colorectal cancer, anti-tumor effect, mitochondrial dysfunction agent, has been the first line regimen for treating CRC over several decades $(2,3)$. However, 5-FU has many disadvantages, for example, poor selectivity and sensitivity to tumor and high toxicity to bone marrow, gastrointestinal tract, and skin even at the therapeutic dose (4). Therefore, combined therapy with 5-FU, named FOLFOX regimen (5-fluorouracil, leucovorin, and oxaliplatin), has widely been used to enhance 5-FU efficiency. However, the problems have been attributed to innate or acquired resistance, resulting from DNA damage repair, decreased drug import or enhanced tolerance to platinum adduct accumulation (5). Therefore, development of new chemotherapeutic agents and strategies are required to improve drug efficacy, tolerance, and disease-free survival.

Parthenolide (PT) is one of the main sesquiterpene lactones present in Feverfew, a traditional herbal medicine, that has been used for the treatment of migraine, fever, and arthritis in Europe (6). It is well known to inhibit interleukin-1 (IL-1) and tumor necrosis factor- $\alpha$-mediated nuclear factor- $\kappa \mathrm{B}(\mathrm{NF}-\kappa \mathrm{B})$ activation, which is responsible for its inflammatory activity (7-9). Recent studies have demonstrated anti-cancer property of PT through induction of apoptotic cell death in a number of human cancer cells $(10,11)$. Multiple pathways might be involved in PT-induced apoptotic cell death, including oxidative stress, endoplasmic reticulum (ER) stress, intracellular thiol depletion, caspase activation, and mitochondrial dysfunction $(12,13)$. Especially, PT has been demonstrated to activate the caspase cascade through regulation of Bcl-2 family, mitochondrial damage, and release of cytochrome $\mathrm{c}$ in cholangiocarcinoma and gastric cancer cells $(11,14)$. Furthermore, it has been shown that the proapoptotic Bcl-2 family (Bid, BAX, BAK) members are important mediators relaying the cell death signaling elicited by PT from caspase 8 downstream in CRC cells (15). Functional role of Bcl-2 family member in PT-induced apoptosis has been studied well. However, there is only one report on PT-induced apoptosis in CRC cells. Especially, PT as a chemotherapeutic agent using a CRC animal model has not been evaluated.

In this study, we examined whether anti-tumor effects of PT involves induction of mitochondrial dysfunction and apoptosis in CRC cell lines and xenograft models. We evaluated the potential as a new chemotherapeutic drug using mouse models of cancer. 


\section{Materials and methods}

Chemicals and reagents. Parthenolide and Z-VAD-FMK were from Calbiochem (San Diego, CA). Rhodamine-123 (Rh-123) and Annexin V-FITC were purchased from Invitrogen (Eugene, OR). TUNEL assay kit was from Promega (Madison, WI). Anti-Bcl-2, anti-Bid, anti-Bax, anti-cytochrome c, anticaspase 3 and anti-p53 antibody were from Santa Cruz (Beverly, MA). Anti-PARP antibody was from Cell Signaling (Beverly, MA). Anti-actin was from Sigma (St. Louis, MO).

Cell culture and treatment. Human colorectal cancer cell lines HT-29, LS174T, and SW480 cells (American Type Culture Collection, Rockville, MD) were cultured in the RPMI-1640 medium supplemented with $10 \%$ FBS, 100 units penicillin and 100 units streptomycin. For the treatment of cells with PT, cells were sub-cultured in RPMI-1640 medium without FBS for $12 \mathrm{~h}$. PT was dissolved in DMSO as a stock solution at $100 \mathrm{mM}$ and diluted with FBS-free medium to achieve designated concentrations. Same concentration of DMSO was always applied to cells as a control.

MTT colorimetric survival assay. HT-29, LS174T, and SW480 cells were plated at a density of $1.0 \times 10^{4}$ cells per well in 96-well plates. Cells were treated with various concentrations of PT for $24 \mathrm{~h}$, and then the medium was removed and $200 \mu \mathrm{l}$ of fresh medium plus $20 \mu \mathrm{l}$ of 3-(4,5-dimethylthiazol-2yl)-2,5-diphenyltetrazolium bromide (MTT, $2.5 \mathrm{mg}$ dissolved in $50 \mu \mathrm{l}$ of dimethylsulfoxide, Sigma) were added to each well. After incubation for $4 \mathrm{~h}$ at $37^{\circ} \mathrm{C}$, the culture medium containing MTT was withdrawn and $200 \mu 1$ of dimethylsulfoxide (DMSO) was added, followed by shaking until the crystals were dissolved. Viable cells were detected by measuring absorbance at $570 \mathrm{~nm}$ using a microplate reader (Molecular Devices, Sunnyvale, CA). The cell growth was expressed as a percentage of absorbance in cells with PT treatment to that in cells without PT treatment $(100 \%)$.

Detection of apoptosis. After being incubated with PT for $24 \mathrm{~h}$, the cells were trypsinized, collected, washed with ice-cold PBS, suspended in a $500 \mu \mathrm{l}$ Annexin $\mathrm{V}$ binding buffer containing $5 \mu \mathrm{l}$ of Annexin V-FITC, and incubated for $15 \mathrm{~min}$ at room temperature in the dark. The fluorescence was measured on a BD LSR flow cytometer (Becton Dickinson, NY) and processed with Cell Quest software (Becton Dickinson, NY) for analysis. PT-induced apoptosis in colon cancer cells was assessed using Hoechst 33258. The cells were treated with various concentrations of PT for $24 \mathrm{~h}$, and then stained with Hoechst 33258 $(1 \mu \mathrm{g} / \mathrm{ml})$ at $37^{\circ} \mathrm{C}$ for $10 \mathrm{~min}$. Nuclear morphology was examined under a Confocal Laser Scanning Microscope (Carl Zeiss, Germany) to identify cells undergoing apoptosis.

Mitochondrial transmembrane potential $(\Delta \Psi m)$. The mitochondrial membrane was monitored using Rhodamine-123 fluorescent dye $(E x / E m=485 \mathrm{~nm} / 535 \mathrm{~nm}$; Sigma $)$, a cell-permeable cationic dye, which preferentially enters into mitochondria due to the highly negative mitochondrial membrane potential $(\Delta \Psi \mathrm{m})$. Depolarization of $\Delta \Psi \mathrm{m}$ results in the loss of Rhodamine-123 from the mitochondria and a decrease in intracellular fluorescence. In brief, cells were incubated with the designated doses of PT for $24 \mathrm{~h}$. Cells were washed twice with PBS and incubated with Rhodamine-123 $(0.1 \mu \mathrm{g} / \mathrm{ml})$ at $37^{\circ} \mathrm{C}$ for $30 \mathrm{~min}$. The intensity of Rhodamine- 123 staining was determined using a BD LSR flow cytometer.

Cell extraction and western blotting. Cells were collected, washed twice with PBS, and then lysed for $30 \mathrm{~min}$ on ice in a lysis buffer (50 mM Tris-HCl pH 8.0, 150 mM EDTA, $1 \%$ Triton $\mathrm{X}-100,0.5 \%$ SDS and protease inhibitor cocktail). The protein concentration in cell lysates was measured by using Protein Quantification kit from Bio-Rad. Total $30 \mu \mathrm{g}$ proteins were loaded onto an SDS-PAGE gel. After transferring and blocking, the membrane was probed with various antibodies (anti-Bcl-2, anti-Bax, anti-Bid, anti-cytochrome c, anti-caspase 3, antiPARP, anti-p53 and anti-actin, Santa Cruz Biotechnology). The signal was detected by using enhanced Westone (Intron, Daejeon, Korea), and captured, analyzed by a Luminescent Image Analyzer (LAS-3000, Fuji film, Japan).

Xenograft models. HT-29 $\left(6 \times 10^{6}\right)$ cells were injected into nude mice. Mice were randomized and assigned to control group and treatment group and intraperitoneally injected 3 times a week vehicle (DMSO) and $4 \mathrm{mg} / \mathrm{kg}$ PT, respectively. PT or vehicle treatment was started on 5 days after tumor cell implantation $\left(0.5 \mathrm{~mm}^{3}\right.$ tumor volume). Tumor diameters were measured 3 times a week, and tumor volumes were also calculated (volume $=\mathrm{X} \times \mathrm{Y} \times \mathrm{Z} \times \pi / 6$ ). The experiment was terminated on 28 days, and the tumors were harvested for immunohistochemistry.

Immunohistochemistry. Immunohistochemistry was carried out in paraffin-embedded $(5 \mu \mathrm{m})$ tissue sections. Apoptosis was measured quantitatively using the terminal deoxynucleotidyl transferase (TdT)-mediated dUTP nick end-labeling (TUNEL) assay using a ApopTag In Situ Apoptosis Detection kit (Chemicon, Temecula, CA) according to the manufacturer's instructions. Four fields at x40 magnification were selected at the proliferation front of each tumor, and TUNEL-positive cells were counted. For analysis of expression of CD31 (VEGF), slides were incubated with anti-CD31 (sc-507; Santa Cruz Biotechnology), and incubated with secondary antibody (goat anti-rabbit; Santa Cruz Biotechnology). Five equal-sized fields were randomly chosen.

Statistical analysis. The data are presented as the mean $\pm \mathrm{SE}$ of at least three independent experiments done in duplicate. Representative blots are shown. All the data were entered into the Microsoft Excel 5.0, and SPSS software was used to perform the two-tailed t-tests or the analysis of the variance, where appropriate. P-values $<0.05$ were considered significant.

\section{Results}

Inhibitory effect of PT on cell proliferation. Human colorectal cancer cell lines, HT-29, SW620, and LS174T cells were treated with various concentrations $(0,5,10,20,40$, and $60 \mu \mathrm{M})$ of PT for $24 \mathrm{~h}$. At $20 \mu \mathrm{M}$ of PT, proliferation of these cells was inhibited approximately $50 \%$ in all cell lines (Fig. 1). At $40 \mu \mathrm{M}$ of PT, inhibition of proliferation of HT-29 and SW620 cells reached over $80 \%$ whereas proliferation of LS174T cells was 


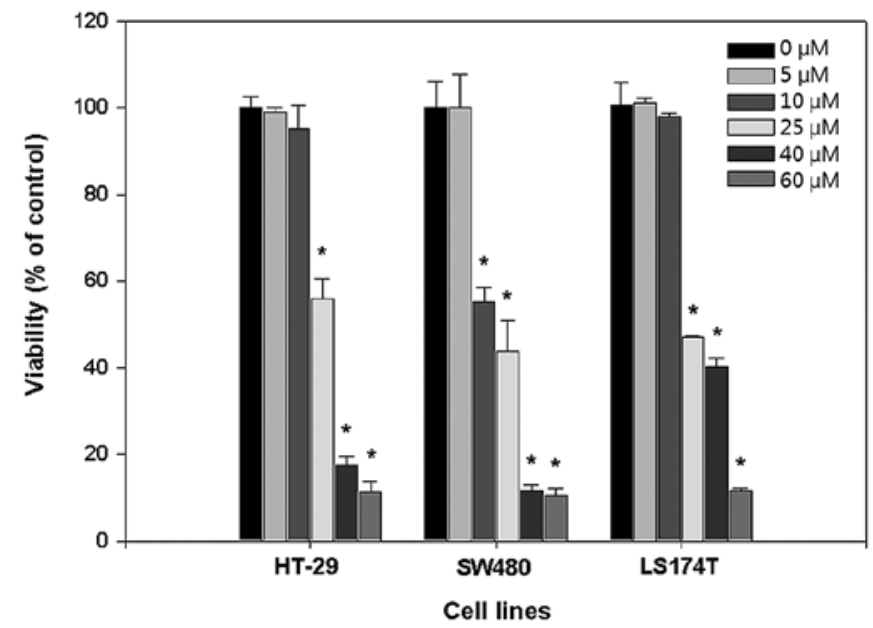

Figure 1. Effect of PT on growth of human colorectal cancer cells. Twenty-four hours of exposure to PT resulted in inhibition of proliferation in a concentration-dependent manner. The experiments were done at least thrice and the result of one representative experiment is shown. Columns, means; bars, $\pm \mathrm{SE}$. ${ }^{*} \mathrm{P}<0.05$ compared with each mean value of HT-29, SW480 or LS174T cells.

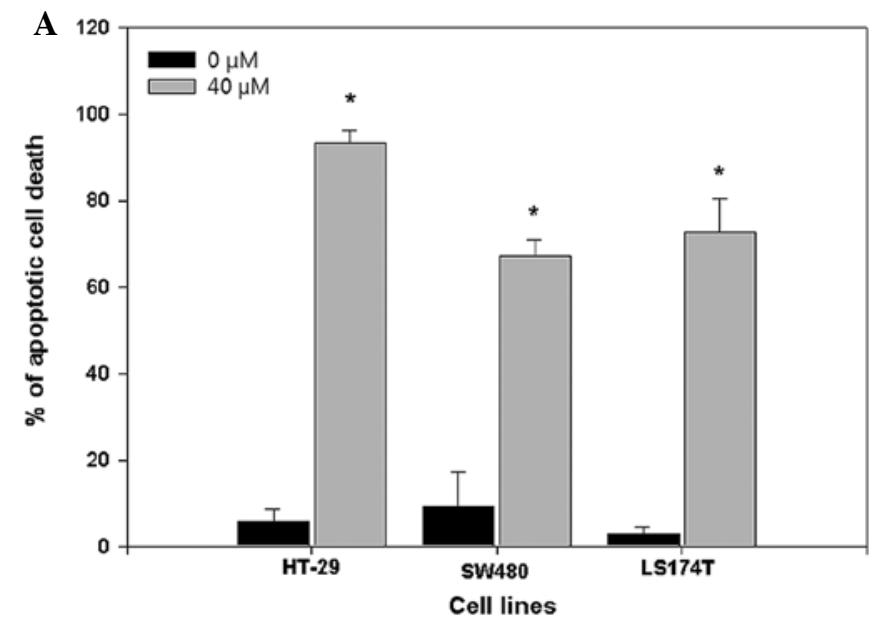

inhibited approximately $60 \%$. At $60 \mu \mathrm{M}$, over $90 \%$ of cell death was observed with all cell lines.

Apoptosis induction by PT. HT-29 cells were treated with 0, 5, 10, 20, $40 \mu \mathrm{M}$ PT for $24 \mathrm{~h}$. The apoptosis of HT-29 cells was induced in a dose-dependent manner of PT, reaching approximately $93.38 \%$ cell death at $40 \mu \mathrm{M}$ of PT. In addition, at $40 \mu \mathrm{M}$ of PT, induction of apoptosis in SW620 and LS174T cells were $67.215 \pm 3.755 \%$ and $72.79 \pm 7.824 \%$, respectively (Fig. 2A).

Apoptotic nuclear morphology was observed after Hoechst 33258 staining using fluorescence microscopy. After treatment with $20 \mu \mathrm{M}$ of PT for $24 \mathrm{~h}, \mathrm{HT}-29$ cells began to exhibit apoptotic characteristics, such as cell shrinkage, nuclear condensation, and fragmentation. At $40 \mu \mathrm{M}$ of PT, DNA condensation was observed in most of cells, and DNA fragments found on surface of glass plate. In the control group, the cells were regular in morphology and grew fully in patches and were confluent, rarely sloughing off (Fig. 2B).

Loss of mitochondrial membrane potential $(\Delta \Psi m)$. HT-29 cells were treated with $0,5,10,20,40 \mu \mathrm{M}$ PT for $24 \mathrm{~h}$. Treatment of the cells with PT caused a decrease in $\Delta \Psi \mathrm{m}$ in HT-29 cells in a PT concentration-dependent manner. The decreased ratio of $\Delta \Psi \mathrm{m}$ peak was $7.29 \%$ (control), $10.36 \%(5 \mu \mathrm{M}), 12.91 \%$ $(10 \mu \mathrm{M}), 13.57 \%(20 \mu \mathrm{M})$ and $57.39 \%(40 \mu \mathrm{M})$ respectively (Fig. 3).

Regulation of Bcl-2 family and cytochrome c release. The underlying mechanism of PT was also explored in the study. The activation of several apoptosis-related proteins may contribute to PT-induced apoptosis. In Bcl-2 family members, the expression of $\mathrm{Bcl}-2$ and truncated-Bid were detected by Western blotting in HT-29. The level of Bcl-2 protein in HT-26 was decreased in a dose-dependent manner of PT (Fig. 4, first panel). In contrast, the expression of truncated-Bid in HT-29

B
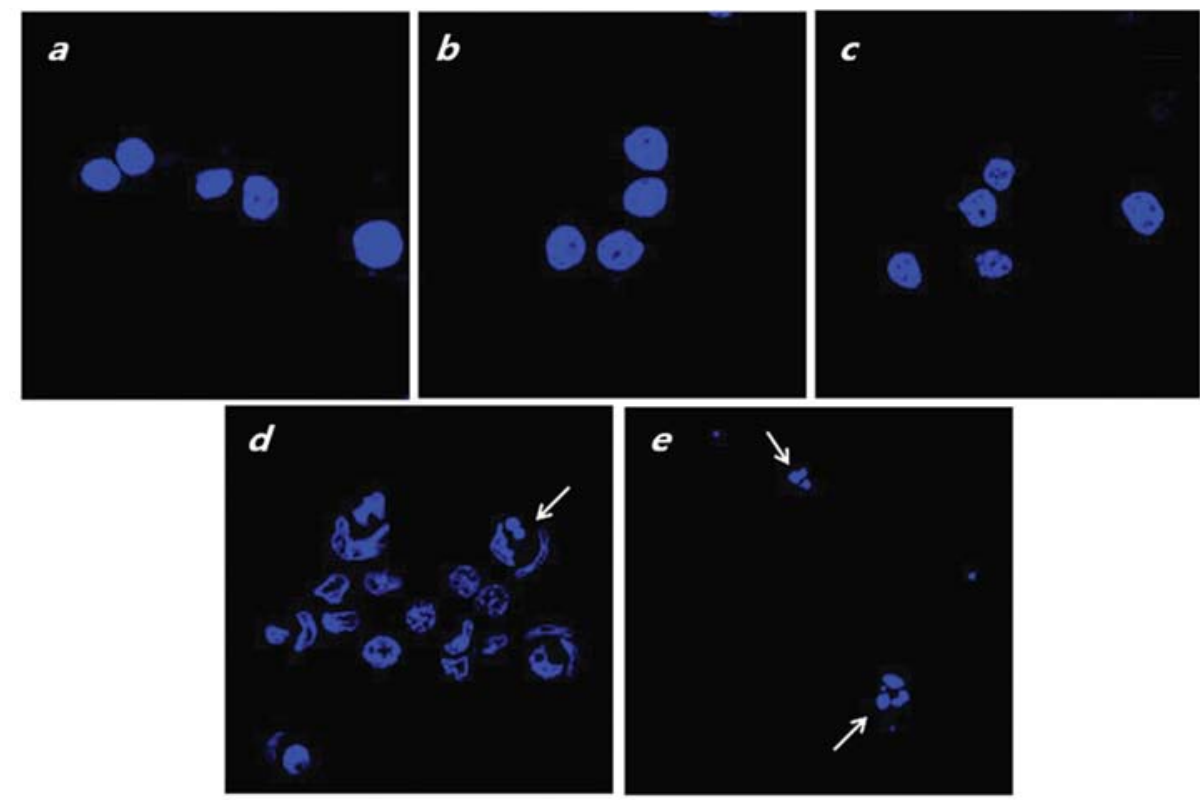

Figure 2. Apoptotic effect of PT on human colorectal cancer cells. (A), FACS analysis of Annexin V-FITC. After PT treatment ( $40 \mu \mathrm{M})$ for $24 \mathrm{~h}$, cells were harvested and stained with Annexin V-FITC. Total 10,000 cells were collected for each group. The experiments were done at least thrice and the result of one representative experiment is shown. Columns, means; bars, \pm SE. "P<0.05 compared with control of HT-29, SW480 or LS174T cells. (B), Hoechst 33258 staining images of the HT-29 cells. After PT treatment (a, control; b, $5 \mu \mathrm{M} ; \mathrm{c}, 10 \mu \mathrm{M} ; \mathrm{d}, 20 \mu \mathrm{M} ; \mathrm{e}, 40 \mu \mathrm{M})$ for $24 \mathrm{~h}$, cells were fixed and stained with Hoechst 33258 (1 $\mu \mathrm{g} / \mathrm{ml})$. 


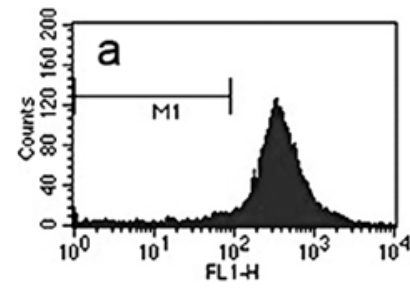

M1 \% of Total : $7.29 \%$

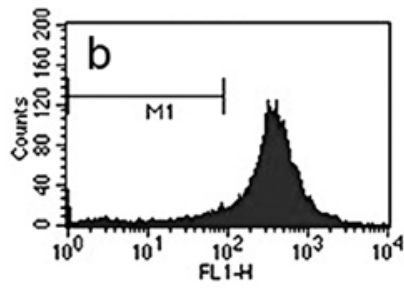

M1 \% of Total : $10.36 \%$

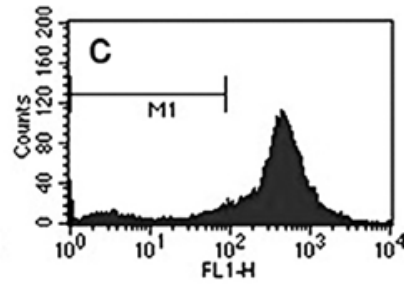

M1 \% of Total : $12.91 \%$

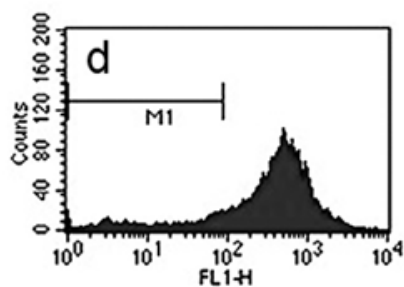

M1 \% of Total : $13.57 \%$

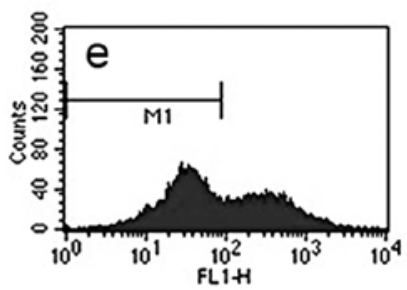

M1 \% of Total : $57.39 \%$

Figure 3. PT-induced changes of mitochondrial membrane potential (MMP). The loss of MMP was measured by Rhodamine-123 (Rh-123) staining and analyzed by flow cytometry. Cells were treated with PT (a, control; b, $5 \mu \mathrm{M} ; \mathrm{c}, 10 \mu \mathrm{M} ; \mathrm{d}, 20 \mu \mathrm{M} ; \mathrm{e}, 40 \mu \mathrm{M}$ ) for $24 \mathrm{~h}$ and then loaded with Rh-123 (5 mg/ml) for $30 \mathrm{~min}$. The inserted histogram demonstrated a left shift of histogram peak representing the decrease of Rh-123 fluorescence intensity due to the loss of MMP. Percentage of cells with reduced fluorescence intensity was calculated.

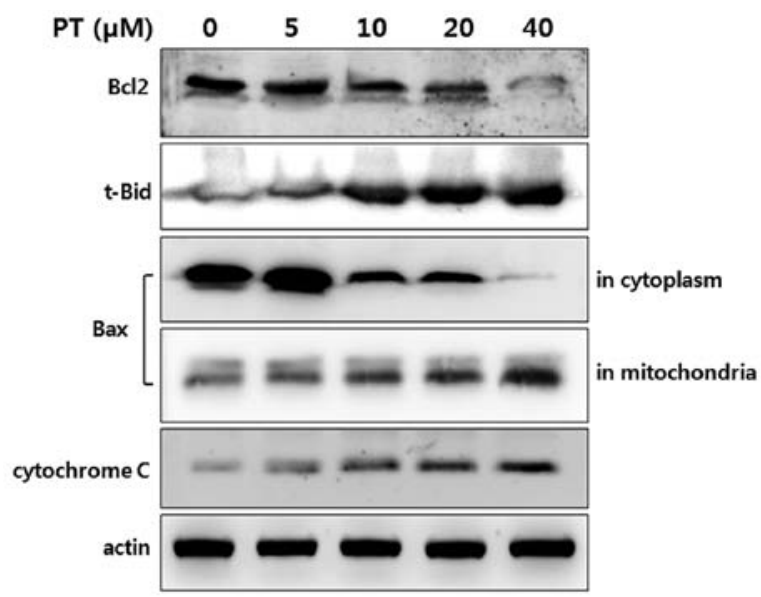

Figure 4. PT-induced regulation of Bcl-2 family member and cytochrome $\mathrm{c}$ release. HT-29 cell was treated with various concentration of PT for $24 \mathrm{~h}$. The total, cytosol and mitochondrial membrane fraction proteins were separated on SDS-PAGE then probed with indicated primary antibody. Actin was used as loading control.

cells was increased dramatically in a dose-dependent manner of PT (Fig. 4, second panel).

Following a mitochondria-dependent death signal, Bax translocates from the cytosol to the mitochondria at which time the Bax conformation changes. This mitochondrial localization of Bax is essential for mitochondrial permeabilization and plays an important role in triggering apoptosis. Therefore, the Bax level was determined in the cytosol and mitochondrial extracts after PT treatment. Treatment of HT-29 cells with PT resulted in a decrease in cytosolic Bax level expression showed a dosedependent progressive decrease of Bax, whereas the Bax level in mitochondrial extracts was increased (Fig. 4, third and fourth panel).

One of the consequences following the changes of Bcl-2 family members is a dissipation of mitochondrial poteintial and release of mitochondrial pro-apoptotic protein, cytochrome c.
A

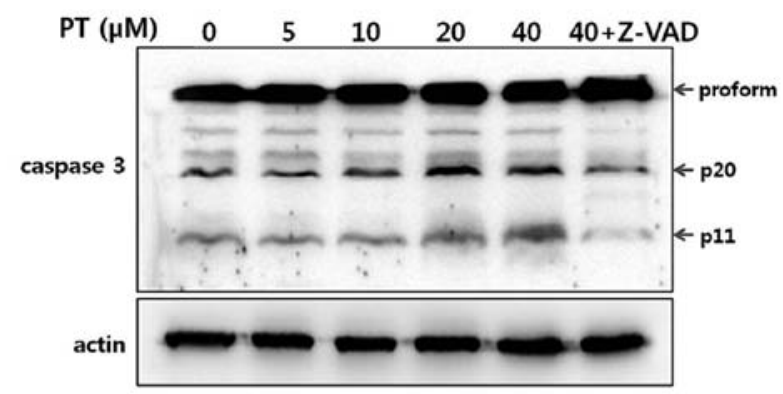

B

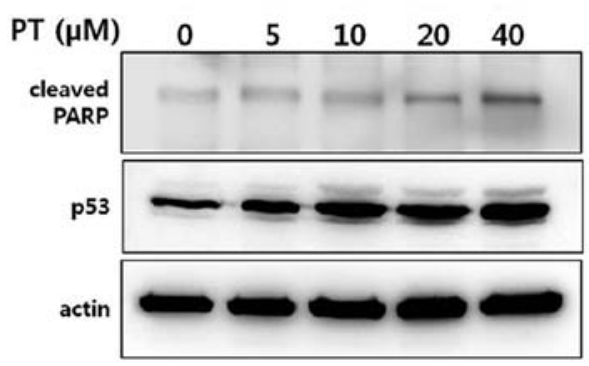

Figure 5.PT-induced activation of caspase 3,PARP cleavage and overexpression of p53. Cells were first pretreated with pan-caspase inhibitor (Z-VAD-FMK), for $1 \mathrm{~h}$, followed by various concentration of PT exposure for $24 \mathrm{~h}$. Then, the total fraction proteins were separated on SDS-PAGE then probed with indicated primary antibody. Actin was used as loading control.

After treatment of HT-29 cells with PT, the release of cytochrome c was increased in a dose-dependent manner (Fig. 4, fifth panel).

Cleavage of caspase 3 and increase of apoptotic marker proteins. Caspase 3 is the terminal factor in the enzymatic cascade reaction related to apoptosis in mammalian cells (16). Here we also tested effects of PT on the caspase 3 activity in HT-29 cells. While amount of pro-caspase 3 was not changed after treatment with PT, cleavage of caspase 3 was increased in a 

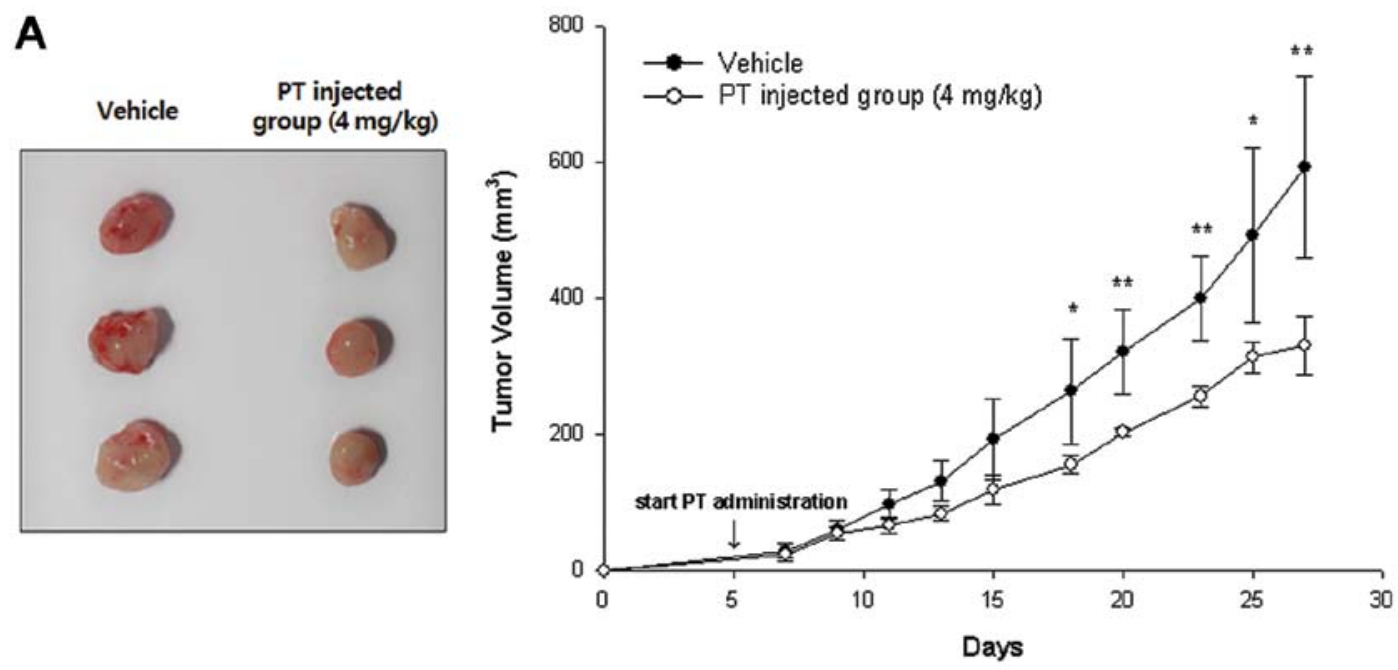

B

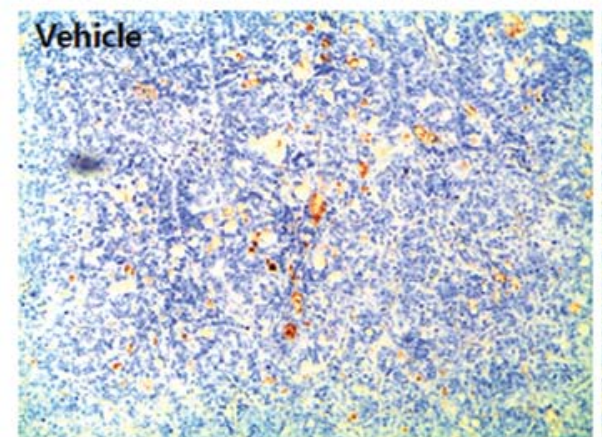

C Vehicle

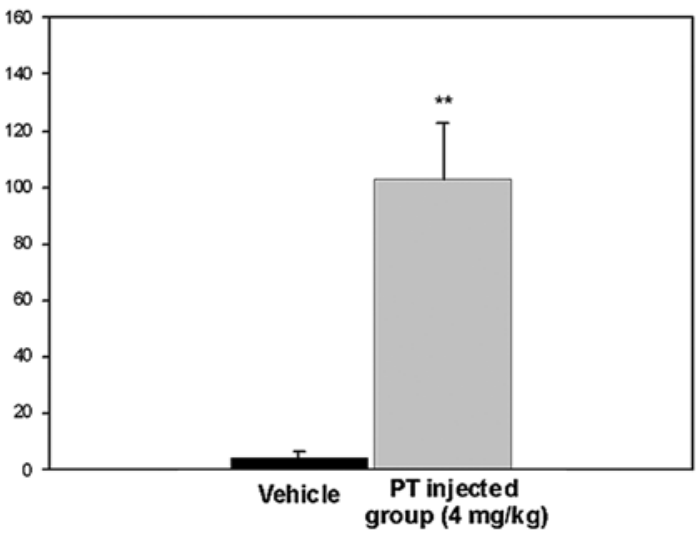

dose-dependent manner. Furthermore, the cleavage of caspase 3 was significantly blocked by pretreatment of a general caspase inhibitor Z-VAD-FMK (Fig. 5A).

The activation of caspase 3 then leads to the cleavage of their downstream molecular targets including PARP and p53, a hallmark of apoptosis $(17,18)$. As shown in Fig. 5B, the levels
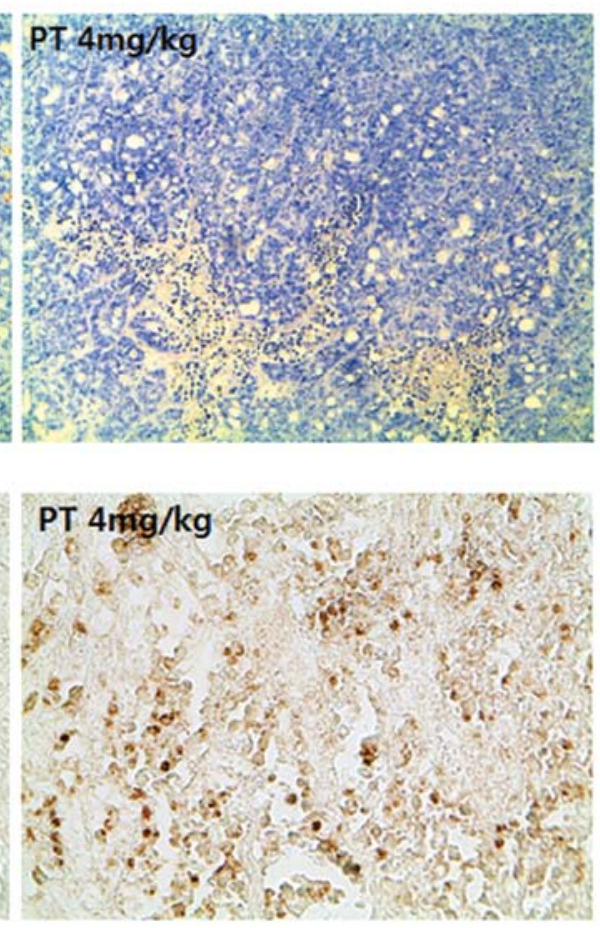

Figure 6. Impact of PT on tumor growth in a subcutaneous xenograft models. (A), Representative image of HT-29 cells xenografts. HT-29 cells were subcutaneously implanted in nude mice. Mice bearing HT-29 cells xenografts were randomized into vehicle and PT treatment groups. Each treatment group consisted of 6-8 mice treated with vehicle, $4 \mathrm{mg} / \mathrm{kg}$ PT for 28 days. Tumor growth was measured and calculated. Points, means; bars, $\pm \mathrm{SE}$. " $\mathrm{P}<0.05,{ }^{* *} \mathrm{P}<0.01$ compared with vehicle group. (B), Tumor sections from animals were fixed and subjected to immunohistochemistry for CD31. Brown spots in image of control mice are positively stained to $\mathrm{CD} 31$. (C), Tumor sections from animals were analyzed by TUNEL assay for apoptotic cell death. The number of apoptotic (TUNEL-positive) cells in tumors was counted in $\times 40$ magnification. Columns, means; bars, $\pm \mathrm{SE} .{ }^{* *} \mathrm{P}<0.01$ compared with vehicle group.

of cleaved PARP and p53 were increased by treatment with PT. Taken together, these results demonstrate that PT-induced apoptosis is caspase 3-dependent (Fig. 5B).

Effect of PT on tumor growth in xenograft mice. To examine the effects of PT on tumor growth in vivo, we used a xenograft 
nude mouse tumor model with subcutaneously implanted HT-29 cells. About 4 weeks after the start of treatment with PT, mean tumor volume of PT-treated mice was $330.01 \pm 42.09 \mathrm{~mm}^{3}$ and it was significantly smaller than that in control mice $\left(592.18 \pm 132.42 \mathrm{~mm}^{3}, \mathrm{p}=0.0075\right.$, Fig. 6A). We further examined the effect of PT on angiogenesis in mice bearing HT-29 cell xenografts. Immunohistochemical analysis of CD31, a wellestablished marker for angiogenesis, revealed that the blood vessel network was well developed in the tumors from control mice, whereas the development of the blood vessel network appeared to be inhibited by PT (Fig. 6B). Representative tumors were also analyzed by the TUNEL assay to determine apoptotic cells. Tumor tissues from mice treated with PT displayed drastically more positively stained apoptotic cells, as compared to those from control mice (Fig. 6C).

\section{Discussion}

Recent reports, including our own, have suggested the involvement of multiple pathways in PT-induced apoptotic cell death in human cancer cells, and the pathways include oxidative stress, inhibition of DNA synthesis, activation of STAT and NF- $\kappa \mathrm{B}$, and mitochodrial dysfunction $(10,11,19-24)$. Especially, PT has been demonstrated to regulate the mitochondrial pathway in vitro. However, there is no report on its ability to inhibit tumor growth in vivo through induction of apoptosis via mitochondrial dysfunction. In the present study, we demonstrated that PT exhibits anti-cancer property, inducing apoptosis via mitochondrial pathway in vitro and in vivo.

Many factors mediating apoptosis converge to activate the critical effector caspase 3, which is considered as the key protease of caspase family in mammalian cell apoptosis (25). Caspase-dependent apoptosis pathway includes mitochondria pathway, death receptor pathway, and endoplasmic reticulum pathway $(26,27)$. The mitochondria pathway is controlled and regulated by the Bcl-2 family $(27,28)$, which are divided into two groups, the anti-apoptotic members (Bcl-2, Bcl-xl) and proapoptotic members (Bax, BAD, Bid) (29). We investigated effects of PT on changes in Bcl-2, truncated-Bid and caspase 3 level. The result of western blot analysis showed that the levels of truncated-Bid and cleaved caspase 3 were increased by treatment with PT, while the level of Bcl-2 was decreased. The cleavage of caspase 3 was prevented by pretreatment with a pancaspase inhibitor, Z-VAD-FMK. These results have suggested that apoptosis induced by $\mathrm{PT}$ involves the caspase-dependent mitochondria pathway. Moreover, another important Bcl-2 family member, Bax is functionally related to truncated-Bid. The direct binding of truncated-Bid to Bax is a prerequisite for Bax translocation into mitochondrial outer membrane, then balance of mitochondrial membrane permeabilization is broken, resulting in cytochrome c release $(30,31)$. In the present study, a dose-dependent progressive decrease of Bax in the cytosol fraction and the increase of Bax in the mitochondrial fraction after PT treatment were detected, suggesting the translocation of Bax from cytosol to the mitochondrial membrane. Through the series of biochemical events, cy tochrome c which is located in the mitochondrial membranes is released into the cytoplasm $(32,33)$. Downstream of cytochrome $\mathrm{c}$, the activation of caspase 3 then leads to the cleavage of their downstream molecular targets including PARP, a hallmark of apoptosis $(17,18)$. Moreover, p53 induces cell cycle arrest or apoptosis in response to DNA damage and regulates Bax and Bcl-2 protein expression (34). In this study, we showed that levels of PARP and p53 were decreased by PT treatment. These observations indicate that PT induces apoptosis via the mitochondrial pathway, causing mitochondrial dysfunction in CRC cells.

Many death signals, such as anti-cancer agents, radiation or ROS could trigger the loss of mitochondrial membrane potential $(\Delta \Psi \mathrm{m})$, a critical step in the apoptosis processes which leads to an irreversible apoptosis (35). Bcl-2 families are mainly involved to apoptosis and mitochondria is the principle site of apoptotic action $(31,36)$. Correspondingly, treatment with $40 \mu \mathrm{M}$ PT induced the $57.39 \%$ of loss of $\Delta \Psi \mathrm{m}$ in HT-29 cells. Notably there were similar changes of Annexin V positive-stained and Rhodamine-123 negative-stained cells in $40 \mu \mathrm{M}$ PT-treated HT-29 cells, suggesting that apoptosis by PT is tightly related to or dependent on the loss of mitochondrial membrane potential $(\Delta \Psi \mathrm{m})$.

To assess the effectiveness of PT against $\mathrm{CRC}$ in vivo, we examined whether PT could inhibit the growth of HT-29 tumors in xenograft models. The growth of HT-29 xenografts was inhibited by treatment with PT and many apoptotic cells were observed in PT treated mice by TUNEL assay. These findings suggest that PT has an effect on tumor growth and apoptosis induction in CRC. The anti-tumor effect of PT by mitochondrial dysfunction has not been studied in vivo. In the present study, we confirm that regulation of Bcl-2 family and dissipation of MMP by PT can lead to inhibit tumor growth in CRC xenografts models.

We also observed reduced blood and blood vessels in PTtreated mice compared to those in control mice. VEGF plays a central role in angiogenesis by promoting the growth of vascular endothelial cells and enhancing vascular permeability $(37,38)$. In a study of renal cell carcinoma, the production of VEGF was decreased by PT in vitro and in vivo (39). Moreover, PT inhibits the proliferation and induces cell cycle arrest at G0/G1 phase in vascular smooth muscle cells (40). Accumulation of these inhibitory effects of PT on angiogenic factors may lead to significant tumor growth inhibition. In this study, we confirm that PT also participates in regulating angiogenesis and suppressing VEGF, using immunohistochemistry images for CD31. Moreover, the regulatory mechanism of angiogenesis by $\mathrm{PT}$ requires further study.

In conclusion, the present study demonstrates that PT inhibits colon cancer development and tumor growth by the induction of apoptosis through mitochondrial dysfunction. These results suggest that PT could be a potential chemopreventive and therapeutic agent of colon cancer.

\section{Acknowledgements}

This study was supported by Fund of Chonbuk National University Hospital Research Institute of Clinical Medicine.

\section{References}

1. Jemal A, Siegel R, Ward E, Hao Y, Xu J and Thun MJ: Cancer statistics, 2009. CA Cancer J Clin 59: 225-249, 2009.

2. Meyerhardt JA and Mayer RJ: Systemic therapy for colorectal cancer. N Engl J Med 352: 476-487, 2005. 
3. Tebbutt NC, Cattell E, Midgley R, Cunningham D and Kerr D Systemic treatment of colorectal cancer. Eur J Cancer 38: 1000-1015, 2002

4. Gusella M, Frigo AC, Bolzonella C, et al: Predictors of survival and toxicity in patients on adjuvant therapy with 5-fluorouracil for colorectal cancer. Br J Cancer 100: 1549-1557, 2009.

5. Raymond E, Faivre S, Chaney S, Woynarowski J and Cvitkovic E: Cellular and molecular pharmacology of oxaliplatin. Mol Cancer Ther 1: 227-235, 2002.

6. Knight DW: Feverfew: chemistry and biological activity. Nat Prod Rep 12: 271-276, 1995.

7. Murphy JJ, Heptinstall S and Mitchell JR: Randomised doubleblind placebo-controlled trial of feverfew in migraine prevention. Lancet 2: 189-192, 1988.

8. Hehner SP, Heinrich M, Bork PM, et al: Sesquiterpene lactones specifically inhibit activation of NF-kappa B by preventing the degradation of I kappa B-alpha and I kappa B-beta. J Biol Chem 273: 1288-1297, 1998

9. Lyss G, Knorre A, Schmidt TJ, Pahl HL and Merfort I: The anti-inflammatory sesquiterpene lactone helenalin inhibits the transcription factor NF-kappaB by directly targeting p65. J Biol Chem 273: 33508-33516, 1998.

10. Zhang S, Ong CN and Shen HM: Critical roles of intracellular thiols and calcium in parthenolide-induced apoptosis in human colorectal cancer cells. Cancer Lett 208: 143-153, 2004

11. Wen J, You KR, Lee SY, Song CH and Kim DG: Oxidative stressmediated apoptosis. The anticancer effect of the sesquiterpene lactone parthenolide. J Biol Chem 277: 38954-38964, 2002.

12. Pajak B, Gajkowska B and Orzechowski A: Molecular basis of parthenolide-dependent proapoptotic activity in cancer cells. Folia Histochem Cytobiol 46: 129-135, 2008.

13. Mathema VB, Koh YS, Thakuri BC and Sillanpaa M: Parthenolide, a sesquiterpene lactone, expresses multiple anti-cancer and antiinflammatory activities. Inflammation 35: 560-565, 2012.

14. Zhao LJ, Xu YH and Li Y: Effect of parthenolide on proliferation and apoptosis in gastric cancer cell line SGC7901. J Dig Dis 10: 172-180, 2009.

15. Zhang S, Ong CN and Shen HM: Involvement of proapoptotic Bcl-2 family members in parthenolide-induced mitochondrial dysfunction and apoptosis. Cancer Lett 211: 175-188, 2004.

16. Chen YC, Shen SC, Lee WR, et al: Emodin induces apoptosis in human promyeloleukemic HL-60 cells accompanied by activation of caspase 3 cascade but independent of reactive oxygen species production. Biochem Pharmacol 64: 1713-1724, 2002.

17. Grutter MG: Caspases: key players in programmed cell death Curr Opin Struct Biol 10: 649-655, 2000.

18. Yang JC and Cortopassi GA: Induction of the mitochondrial permeability transition causes release of the apoptogenic factor cytochrome c. Free Radic Biol Med 24: 624-631, 1998.

19. Tacchini L, De Ponti C, Matteucci E, Follis R and Desiderio MA Hepatocyte grow th factor-activated NF-kappaB regulates HIF-1 activity and ODC expression, implicated in survival, differently in different carcinoma cell lines. Carcinogenesis 25: 2089-2100, 2004.

20. Dai Y, Guzman ML, Chen S, et al: The NF (nuclear factor)kappaB inhibitor parthenolide interacts with histone deacetylase inhibitors to induce MKK 7/JNK1-dependent apoptosis in human acute myeloid leukaemia cells. Br J Haematol 151: 70-83, 2010.

21. Nakshatri H, Rice SE and Bhat-Nakshatri P: Antitumor agent parthenolide reverses resistance of breast cancer cells to tumor necrosis factor-related apoptosis-inducing ligand through sustained activation of c-Jun N-terminal kinase. Oncogene 23 7330-7344, 2004
22. Carlisi D, D'Anneo A, Angileri L, et al: Parthenolide sensitizes hepatocellular carcinoma cells to TRAIL by inducing the expression of death receptors through inhibition of STAT3 activation. J Cell Physiol 226: 1632-1641, 2011.

23. Chen KF, Tai WT, Liu TH, et al: Sorafenib overcomes TRAIL resistance of hepatocellular carcinoma cells through the inhibition of STAT3. Clin Cancer Res 16: 5189-5199, 2010.

24. Wu C, Chen F, Rushing JW, et al: Antiproliferative activities of parthenolide and golden feverfew extract against three human cancer cell lines. J Med Food 9: 55-61, 2006.

25. Fernandes-Alnemri T, Litwack G and Alnemri ES: CPP32, a novel human apoptotic protein with homology to Caenorhabditis elegans cell death protein Ced-3 and mammalian interleukin-1 beta-converting enzyme. J Biol Chem 269: 30761-30764, 1994.

26. Mehmet H: Caspases find a new place to hide. Nature 403: 29-30, 2000.

27. Yang E and Korsmeyer SJ: Molecular thanatopsis: a discourse on the BCL2 family and cell death. Blood 88: 386-401, 1996.

28. Green DR and Reed JC: Mitochondria and apoptosis. Science 281: 1309-1312, 1998.

29. Reed JC: Double identity for proteins of the Bcl-2 family. Nature 387: 773-776, 1997.

30. Adams JM and Cory S: The Bcl-2 protein family: arbiters of cell survival. Science 281: 1322-1326, 1998

31. Gross A, McDonnell JM and Korsmeyer SJ: BCL-2 family members and the mitochondria in apoptosis. Genes Dev 13: 1899-1911, 1999.

32. Terrones O, Antonsson B, Yamaguchi $\mathrm{H}$, et al: Lipidic pore formation by the concerted action of proapoptotic BAX and tBID. J Biol Chem 279: 30081-30091, 2004.

33. Van Mau N, Kajava AV, Bonfils C, Martinou JC and Harricane MC: Interactions of Bax and tBid with lipid monolayers. J Membr Biol 207: 1-9, 2005.

34. Coutts AS and La Thangue N: The 553 response during DNA damage: impact of transcriptional cofactors. Biochem Soc Symp pp181-189, 2006.

35. Kroemer G and Reed JC: Mitochondrial control of cell death. Nat Med 6: 513-519, 2000.

36. Borner C: The Bcl-2 protein family: sensors and checkpoints for life-or-death decisions. Mol Immunol 39: 615-647, 2003.

37. Ferrara N, Hillan KJ, Gerber HP and Novotny W: Discovery and development of bevacizumab, an anti-VEGF antibody for treating cancer. Nat Rev Drug Discov 3: 391-400, 2004.

38. Huang S, Pettaway CA, Uehara H, Bucana CD and Fidler IJ: Blockade of NF-kappaB activity in human prostate cancer cells is associated with suppression of angiogenesis, invasion, and metastasis. Oncogene 20: 4188-4197, 2001.

39. Oka D, Nishimura K, Shiba M, et al: Sesquiterpene lactone parthenolide suppresses tumor growth in a xenograft model of renal cell carcinoma by inhibiting the activation of NF-kappaB. Int J Cancer 120: 2576-2581, 2007.

40. Weng SX, Sui MH, Chen S, et al: Parthenolide inhibits proliferation of vascular smooth muscle cells through induction of G0/ G1 phase cell cycle arrest. J Zhejiang Univ Sci B 10: 528-535, 2009. 